UC-21

- Issued: March 1983

LA--9540-MS

DE83 009785

\title{
Vacuum Laser-Initiated Plasma Shutters for Retropulse Isolation in Antares
}

T. W. Sheheen

S. J. Czuchlewski

J. Hyde

R. L. Ainsworth

\section{DSSCLAIMITR}

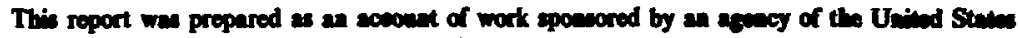

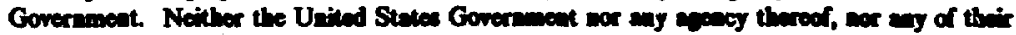

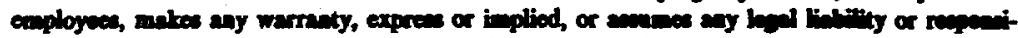

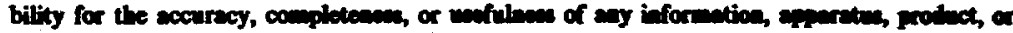

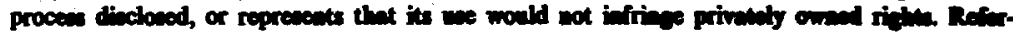

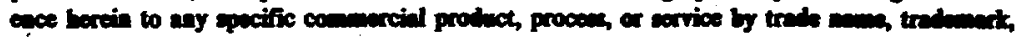

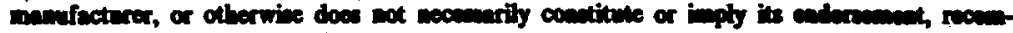

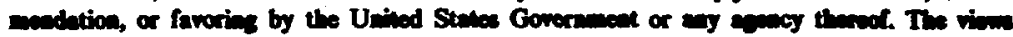

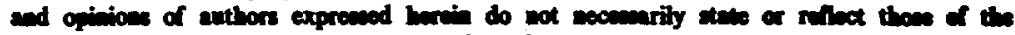
United Stutes Government or any eneacy thenof. 


\title{
VACUUM LASER-INITIATED PLASMA SHUTTERS FOR RETROPULSE ISOLATION IN ANTARES
}

by

T. W. Sheheen, S. J. Czuchlewski, J. Hyde, and R. L. Anworth

\begin{abstract}
We have demonatrated that sintered LF spatial fiters may be used in a $10^{-6}$-torr vacuum environment as laser-initiated plasm suutters for retropula ivolation in the Antares high-enercy laser-fivion system. In our experiments, a 1.1-ms pulved $\mathrm{CO}_{2}$ laser, at a 10 - $\mu \mathrm{m}$ wavelength and an energy of up to $3.0 \mathrm{~J}$, was ued for plasm imitiation; a chopped probe laser tuned to a $9.6-\mu \mathrm{m}$ wavelength was used in determinimg the blockins

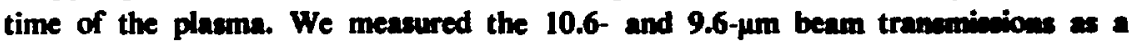
function of fluence on the aperture edze. For an 800- $\mu \mathrm{m}$-diam aperture and a 1.2-mmdiam Gaussian beam determined at the $1 / e^{2}$ intencity points, we obwerved blockins times in excess of $1.0 \mu \mathrm{s}$.
\end{abstract}

\section{INTRODUCTION}

In all high-energy laser fusion systems, protection of front-end optical components from damage by the laser pulses reflected into the laser system from fusion targets is important. Any apparatus used in isolating the retropulse should have a high forward transmission and a low backward transmission; be minimally complex for reliable operation; and for the Antares configuration, function in a vacuum environment.

Although an exploding wire system ${ }^{1}$ and a metal iris in air or ambient nitrogen ${ }^{2}$ can block target retro-reflected light, none of these retropulse-isolation approaches seem appropriate for Antares. The exploding wire method is not cost efractive, and the vacuum environment of Antares is not compatible with a metal iris in ambient gas. Even though experiments have been performed using a metal iris in vacuum in which a vacuum-blocking plasma was formed, ${ }^{3}$ this device suffers from prompt damage to the pinhole at fluences of $240 \mathrm{~J} / \mathrm{cm}^{2}$ or above, without providing a critical-density plasma with the lifetime of $\mathbf{5 3 0}$ ns or greater required for the Antares beamline configuration.

In this report we demonstrate that $\& \mathrm{CO}_{2}$ laser $\approx 10.6$ $\mu \mathrm{m}$ can initiate a plasma at the aperture of a sintered LiF spatial filter in a $10^{-6}$-torr vacuum that will be of sufficient density and lifetime to block the retropulse from re-entering the laser system. The fluence incident on the edge of the spatial-filter aperture controls the plasmainitiated process and retropulse-blocking time. With the 1.1-ns pulsed 10.6- $\mu \mathrm{m} \mathrm{CO}_{2}$ laser incident on the edre of a $0.787-\mathrm{mm}$-diam LiF spatial-fitter aperture a a 63 $\mathrm{J} / \mathrm{cm}^{2}$ fluence level, the Iaser-initiated plasma transmits 64\% of the incident beam within the 1.1-ns pulue width. Within $40 \mathrm{~ns}$, the plasma reaches a critical density of

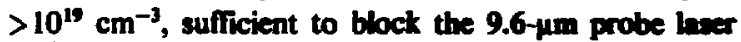
for $680 \mathrm{~ns}$. At greater edge fluences, blocking tines in excess of $1.0 \mu$ may be obtainad for the 9.6-pm probe laser. Athough we obeerved conaiderable danne to the metal iris in vecuum at high flence bevels, the simened LiF spatial filter shows only niminiel damage, willow distortion of the aperture, after more then 200 perises in a $10^{-6}$-torr vacuum. 


\section{EXPERIMENTAL TECHNIQUE}

\section{A. Experimental Arrangement}

Our experiments employ a 10.6- $\mu \mathrm{m} \mathrm{CO}_{2}$ laser with a beam diameter of $3.0 \mathrm{~cm}$, a pulse width of $1.1 \mathrm{~ns}$, and an energy of up to $3.0 \mathrm{~J}$ per pulse. This laser is focused on the LiF spatial filter in a $10^{-6}$-torr vacuum through an $\mathrm{NaCl}$ window and lens with a $60-\mathrm{cm}$ focal length for an $\mathrm{f} / 20$ system. We used a chopped $\mathrm{ew} \mathrm{CO}_{2}$ laser tuned to 9.6 $\mu \mathrm{m}$ as a probe laser to determine blocking time of the laser-initiated plasma. The beam paths and experimental configuration are shown schematically in Fig. 1 .

Pyroelectric joulemeters measured the 10.6- $\mu \mathrm{m}$ laser input to the spatial filter and the transmitted energy. Inserting calibrated $\mathrm{CaF}_{2}$ attenuators varied output without changing the input beam diameter. The spatial filter was aligned for maximum transmission using the micrometer adjustments that are fed through the vacuum chamber wall. A video system consisting of a lens, telescope, camera, and monitor is used during alignment. It is calibrated for beam/spatial-filter position so that any misalignment or asymmetry that develops during plasma formation can be detected on subsequent realignment. We aligned the 9.6- $\mu \mathrm{m}$ probe laser with the spatial filter by adjusting the two copper mirrors in the vacuum chamber for maximum signal on the liquid-nitrogencooled ( $\left.\mathrm{Hg}_{\mathrm{g}} \mathrm{Cd} \mathrm{Te}\right) \mathrm{SAT}$ detector.

\section{B. Beam Profile Measurements}

Using three methods, we determined the profile of the 10.6- $\mu \mathrm{m}$ beam with an attenuated beam: (1) burn patterns on exposed Polaroid film in and out of the focal piane; (2) a $0.190-\mathrm{mm}$ pinhole scan in front of a pyroetectric joulemeter; and (3) as a primary diagnostic, direct measurement of the beam pirofile with a 32 element Sprincon pyroelectric linear array having 0.10 by $0.0875-\mathrm{mm}$ ejements on $0.10-\mathrm{mm}$ centers. Approximately Gaussian profile data were taken in the vertical and horizontal positions in and out of the focal plane and recorded using a Tektronix 7834 scope. The methods used in determining the beam profile and position are

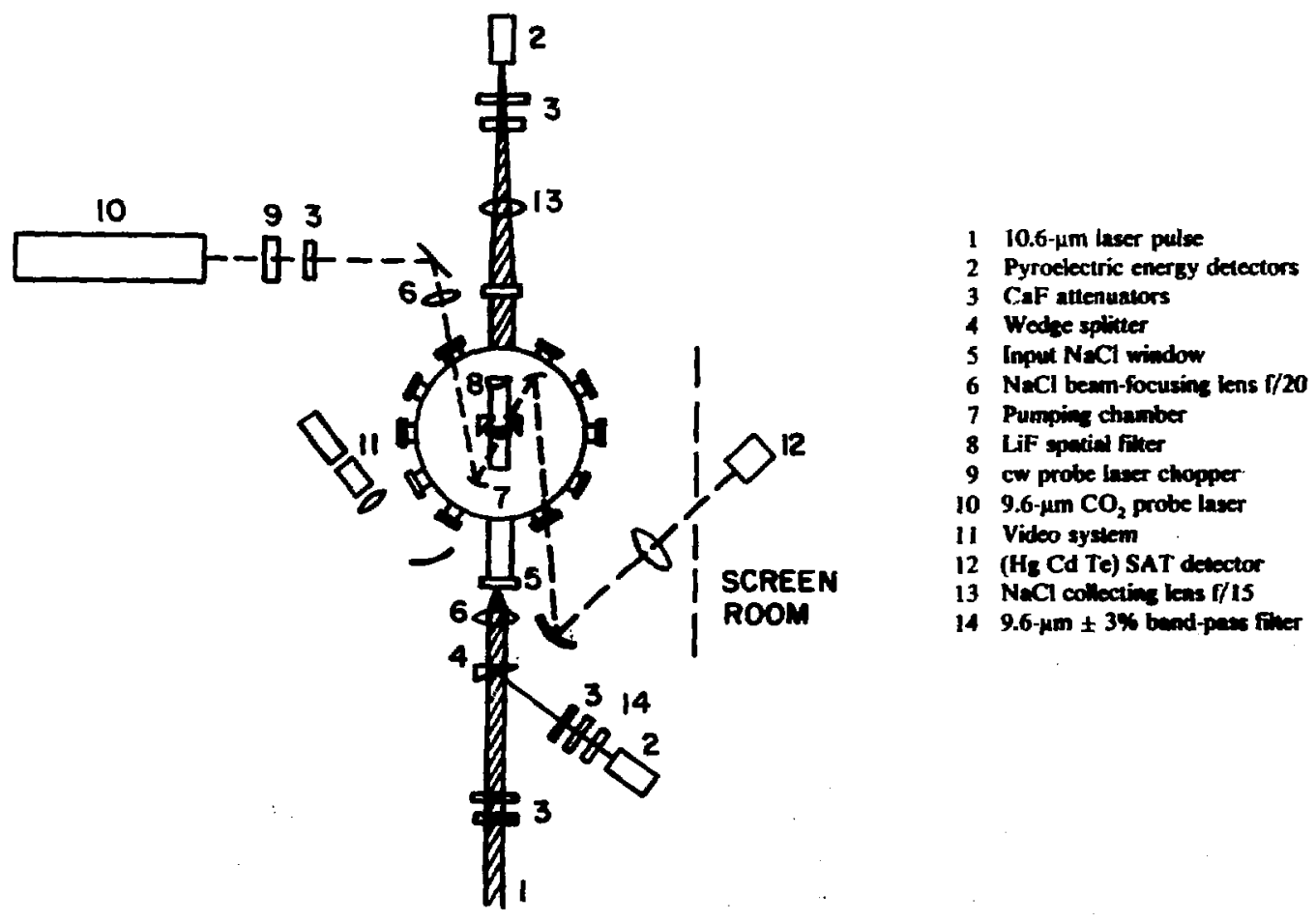

Fis. 1. Schematic of beam paths and experimental configuration. 
within $\pm 20 \%$ agreement; also, shot-to-shot variation was determined with the linear array and is within $\pm 20 \%$ agreement.

\section{Spatial-Filter Dexign}

As shown in Fig. 2, a paraxial ray at an angle $\alpha$ off axis, entering a spatial filter with a cone half angle of $\phi$, is reflected at an angle $\pi_{1}$, measured from the normal to the conical surface, given by

$\pi_{1}=90^{\circ}-\phi+\alpha$.

Succeeding reflections are at angles $\pi_{n}$,

$\pi_{n}=v_{n-1}-2 \phi$,

for $\mathrm{n}=2,3,4, \ldots$ As long as $\pi_{n}>0$, propagation continues toward the apex of the cone.

In our experiments the spatial filter has $\phi=39.4^{\circ}$, and we employ a converging beam having rays with $0 \leq \alpha \leq$ $1.4^{\circ}$. Then $50.6^{\circ} \leq \pi_{1} \leq 52.0^{\circ}$, and $-28.2 \leq \pi_{2} \leq-26.8$; whence the beam exits the cone after the second reflection.

The spatial filter for Antares has a cone angle $\phi$ of $18.2^{\circ}$, and the incoming beam has a convergence half angie of $1.0^{\circ}$. The first few reflection angles will then be

$71.8^{\circ} \leq \pi_{1} \leq 72.8^{\circ}$,

$35.4 \leq \pi_{2} \leq 36.4$,

$12.0 \leq \pi_{3} \leq 0$,

and

$-37.4 \leq \pi_{4} \leq-36.4$.
The third reflection leaves the surface normally, and subsequent reflections move back out of the cone.

\section{EXPERIMENTAL RESULTS}

The $\mathrm{CO}_{2}$ laser probe beam transmianion is detected using a liquid-nitrogen-cooled ( $\mathrm{H}_{\boldsymbol{g}} \mathrm{Cd}$ Te) SAT detector located in a screen room with a fast storase scope for data recording. The blocking time by the spatial fitter sperture (Fig. 3) is a function of the 10.6- $\mu \mathrm{m}$ fluence on the aperture edze. A 1-mm quartz-core fiber optic was used on each data shok to carry light from the plasma to an RCA C 3080F PIN photodiode biased at $+30 \mathrm{~V}$, and the pulse width of the signal agrees with the blocking times shown in Fig. 3.

Figure 4 shows transmission of the 1.1-ns pulsed $\mathrm{CO}_{2}$ laser versus fluence incident on the edge of the spatialfilter aperture. The dashed curve merely highlights the higher transmission at a fluence of approximately $\mathbf{1 0 . 7}$ $\mathrm{J} / \mathrm{cm}^{2}$ on the aperture edge. The effect of a higher transmission may be real because the same behavior has been noted using a metal iris in a nitrogen environment; 2 we think this may represent a plasma lens effect. ${ }^{3}$ Also indicated is a plasma closure time of less than the 1.1-ns pulse width of the 10.6- $\mu \mathrm{m}$ laser pulse.

The blocking time of the $\mathrm{cw}$ probe laser at $9.6 \mu \mathrm{m}$ versus the percentage of transmistion of the I.1-ns pulsed $\mathrm{CO}_{2}$ laser at $10.6 \mu \mathrm{m}$ (Fig. 5) agrees with the data in Figs. 3 and 4. Figure 6 shows transmitted energy of the 1.1-ns pulsed laser through the spatial filter as a function of aperture edge-fluence incident and also represents E-out versus $E$-in for this beam and aperture geometry; we did not observe any clampins of the transmitted beam.

Figure 7 shows the blocking time of the probe laser as a function of total fluence incident on the spatial finer

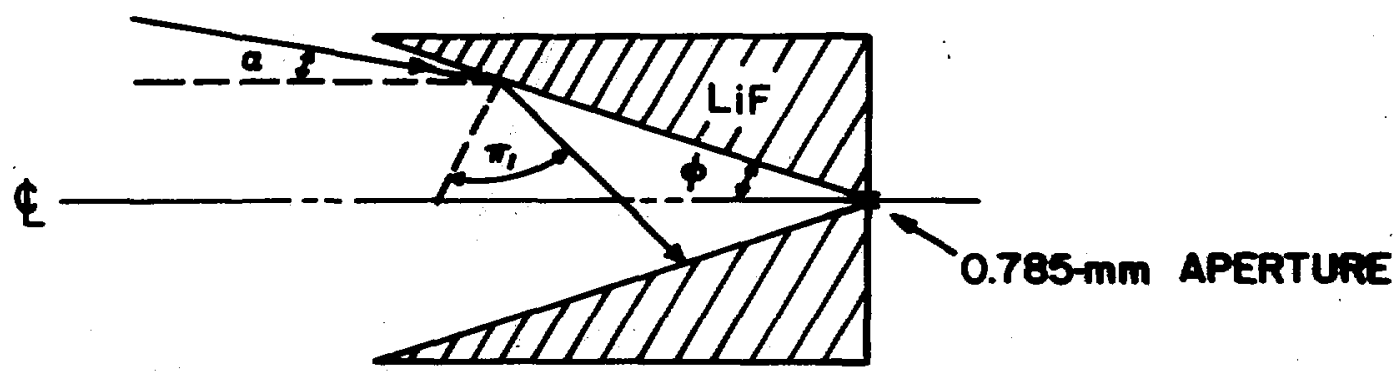

F2. 2. Antures LiF spocial Tiver. 


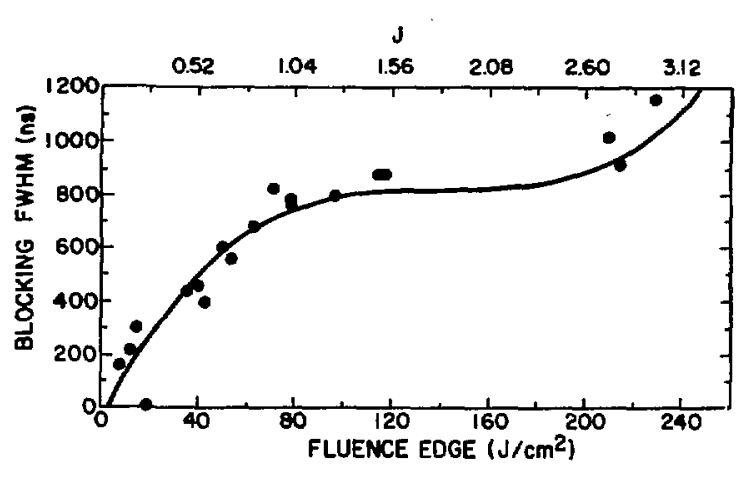

Fig. 3. Blocking time versus edge fluence.

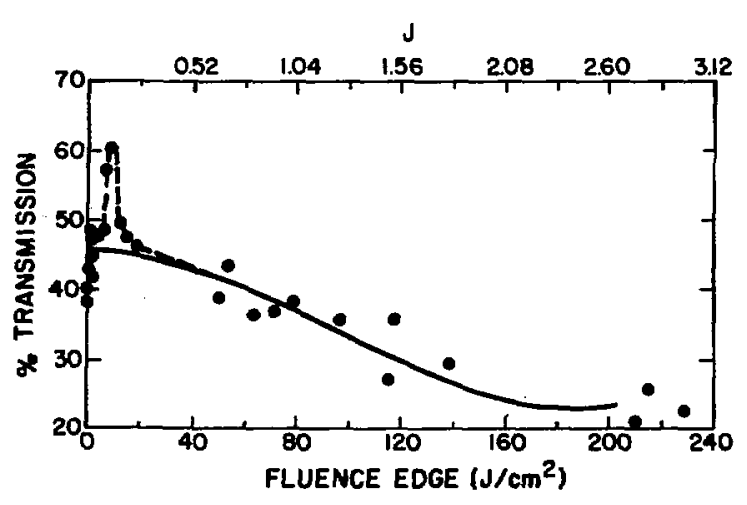

Fig. 4. Per cent transmission versus edge fuence.

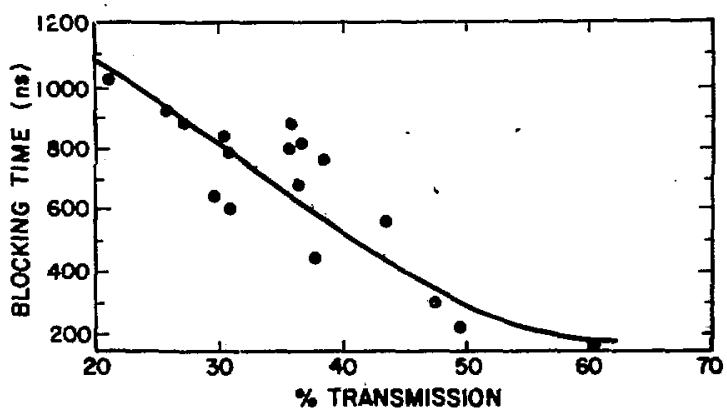

Fig. 5. Blocking time versus per cent transmission.

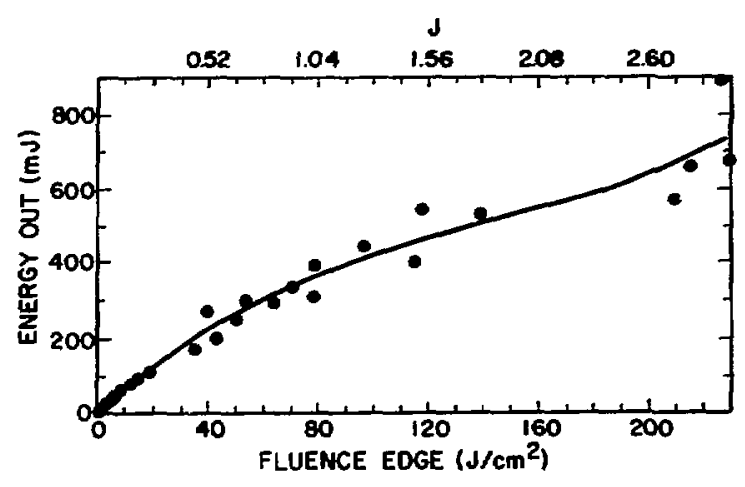

Fig. 6. Energy out versus edge fuence.

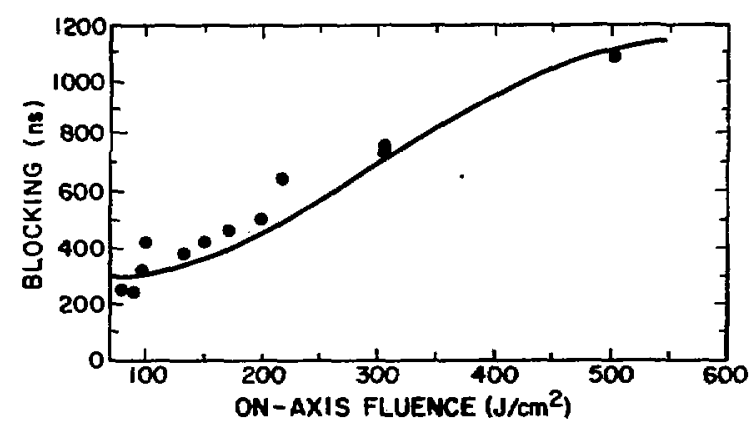

Fig. 7. Blocking time versus on-axis fuence.

with the pulsed $\mathrm{CO}_{2}$ laser focused $2.0 \mathrm{~mm}$ off the spatialfilter axis. Blocking of the probe laser does occur but at higher fluence levels. During the test series, we exposed the LiF spatial filter to 200 laser pulses in a vacuum environment without distorting or opening the aperture. Sputtering occurred around the circumference of the aperture where the pulsed beam is incident. A 650$\mu \mathrm{m}$-diam metal iris was initially tried in vacuum at $10^{-6}$ torr at the same $\mathrm{CO}_{2}$ beam intensity: and was noticeably damaged after $\sim 50$ pulses. However we. believe that further work is required to entablish more definitive useful lifetimes.

Statistical fluctuations of $\pm 20 \%$ in transmiscion values were observed. These are probably due to the somewhat erratic nature of the plasma initiotion process. An uncertainty of a factor of 2 is preseat in the absolute values of the odve flences, which is camed by the uncertainties in meanuring the bean profiles and in aligning the beam on the aperture. The macrionity in the relative fluence values is much betier, homever, being $\pm 10 \%$. 


\section{DISCUSSION}

\section{A. Experimental Deta}

In interpreting our experimental results, a primary consideration is the spatial profile of the $10.6-\mu \mathrm{m}$ puleed laser beam. The experimentally determined spatial profile was approximately Gaussian, with a radius defined at the $1 / \mathrm{e}^{2}$ intensity points of $540 \pm 75 \mu \mathrm{m}$, at a position 20.0 $\mathrm{mm}$ in front of the $\mathrm{f} / \mathbf{2 0}$ input optics focal plane, where the spatial-filter aperture was positioned to obtain edge fluences of interest.

The theoretical beam radius at the $1 / e^{2}$ point for a perfect optical system at a distance $z$ in front of the focal plane is given by

$\omega_{z}=\omega_{0}\left[1+\left(\frac{\lambda z}{\pi \omega_{0}^{2}}\right)^{2}\right]^{1 / 2}$.

In this expression, $\omega_{0}$ is the radius at the $1 / e^{2}$ point in the focal plane and is defined as

$\omega_{0}=\frac{2.0 \lambda \mathrm{f}_{\#}}{\pi}$,

where $f \#$ is the $f /$ number of the optical system.

To determine whether the experimental results for the pulsed 10.6- $\mu \mathrm{m}$ laser beam are self-consistent, we constructed an "idealized" Gaussian beam profile from the experimental data. By assuming a $3.067-\mathrm{cm}$ input beam diameter and a $23.29-\mathrm{mm}$ spatial-filter aperture position $z$ in front of the focal plane, we could define a beam with a pure Gaussian spatial profile that would fit the experimental data well within the $20 \%$ experimental error. Using the $3.067-\mathrm{cm}$ input beam diameter with the $60.0-\mathrm{cm}$ focal length $\mathrm{NaCl}$ input lens, we have an $\mathrm{f} / 19.56$ input optics system and a focal plane beam radius $\omega_{0}$ of $132 \mu \mathrm{m}$. The beam radius $\omega_{2}, 23.29 \mathrm{~mm}$ in front of the focal plane, is then $610 \mu \mathrm{m}$. For such a beam, the maximum transmission of the 10.6- $\mu \mathrm{m}$ laser beam through the spatial filter with a $0.3937-\mathrm{mm}$ aperture radius is $56.53 \%$ of the total energy input $\left(E_{1}\right)$ on the filter axis. For this we use

$$
E_{\text {out }}=E_{(n)}=\frac{\pi \omega_{z}^{2}}{2} F_{0}\left[1-\exp \left(\frac{-2 r^{2}}{\omega_{z}^{2}}\right)\right] \text {, }
$$

with the peak on-axis fluesce Fo given by

Fo $=\frac{2.0 \mathrm{E}_{\mathrm{m}}}{\pi \omega_{2}^{2}}$

Note that, at $m$ edge fuence between 6 and $10 \mathrm{~J} / \mathrm{cm}^{2}$, the transmistion of the pulsed 10.6-pm $\mathrm{CO}_{3}$ beam exceeds the maximum calculated or smal-signal tranemission. Czuchlewski and Figueira ${ }^{2}$ noticed this eflect when a metal iris was used in a nitrogen-gas experiment. We conjecture that a subcritical plarma (with a density of less than $10^{19} \mathrm{~cm}^{-3}$ for the $10.6-\mu \mathrm{m}$ radiation) is formed, with an index of refraction given by

$n=\left(1-\frac{\omega_{0}^{2}}{\omega^{2}}\right)^{1 / 2}$,

where $\omega_{p}$ is the plasma frequency and 0 is the optical frequency. The formation occurs in less time than that of the 1.1-ns laser pulse width; thus, the plasma acts as a positive lens, "focusing the pulsed laser beam through the aperture of the spatial filter into the $f / 15$ collecting optics.

The data in Fig. 6 show that the laser-initiated plasma is formed within the 1.1-ns time scale of the inser plasma pulse with a density sufficient to decrease the transmission of the plasma-initiating laser pulse. However, at the fluences used in these experiments, the 10.6- $\mu \mathrm{m}$ laser pulse is not self-clamped, ${ }^{2}$ to the point that the transmission through the spatial fiker appronches zero. This situation is most clearly shown in Fig. 6, where a plateau is absent in the energy transmitted as a function of the odge fluence.

The time during which the 9.6- $\mu \mathrm{m}$ probe laser beam transmission is blocked is a function of the pubed 10.6$\mu \mathrm{m}$ laser fluence on the spativl-fiter aperture edge (Fiz. 3). At fluences of $230 \mathrm{~J} / \mathrm{cm}^{2}$, blocking times for the probe laser are greater then $1.1 \mathrm{\mu s}$, a time that exceeds the reflection time of the laser pulse from a fusion target in the Antares laser fusion system. The plasen formation time needed to reach critical density suficiont to block the 9.6- $\mu \mathrm{m}$ beam is $\sim 40 \mathrm{~ms}$. The mochanim by which the plasma reaches this density is not decernible from our present experimental data. Howewer, we speculate that as the LiF is blown of the spovid ther the bingom

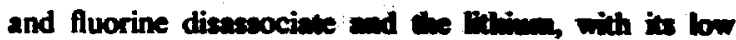
work function, is readily ionized by the and atic elec-

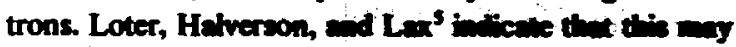


be a plausible mechanism; they focused a $10.6-\mu \mathrm{m} \mathrm{CO}_{2}$ laser with a $3 \times 10^{11}-\mathrm{W} / \mathrm{cm}^{2}$ peak irradiance on a $\mathrm{CF}_{2}$ target in soft vacuum and observed $F$ VIII lines at $16.807 \dot{A}$. This flux is comparable to the peak irradiance in our experiment of $2.33 \times 10^{11} \mathrm{~W} / \mathrm{cm}^{2}$.

From the experimental data, we cannot explain the plateau in blocking time at approximately $800 \mathrm{~ns}$, but we think it is a region where plasma formation is in equilibrium with losses. A similar situation is shown in Fig. 7: the blocking time of the $9.6-\mu \mathrm{m}$ probe beam as a function of total fluence approaches an asymptotic value of approximately $1.1 \mu \mathrm{s}$ when the pulsed $10.6-\mu \mathrm{m}$ beam is focused $2.0 \mathrm{~mm}$ off the spatial-filter axis. With the pulsed beam focused off-axis, the angle of incidence on the LiF surface is $39^{\circ}$, which gives a reflectance per steradian of $0.004 . *$ This result indicates that most of the

* This information was supplied by A. Saxman, Group P-5, Los Alamos National Laboratory, in "Geometry for Scattering and Reflection Measurements" (1982). laser energy is deposited on the surface of the spatial filter at first incidence.

Plasma lifetimes, determined by using fiber-optics probe diagnostics to detect the plasma radiation, agree very well with the blocking times observed for the probe laser. This agreement serves as an independent correlation of the plasma lifetime with the critical density necessary to block the $9.6-\mu \mathrm{m}$ beam by the laser-initiated plasma. The observed luminosity is probably due to bremsstrahlung ${ }^{6}$ that is proportional to electron density because the plasma should be at a temperature too high for significant recombination.

\section{B. Retropulse Isolation in Antares}

The optical beam path and elements of the Antares power amplifier are shown schematically in Figs. 8 and 9; the element spacings are shown in Fig. 9. A plasma

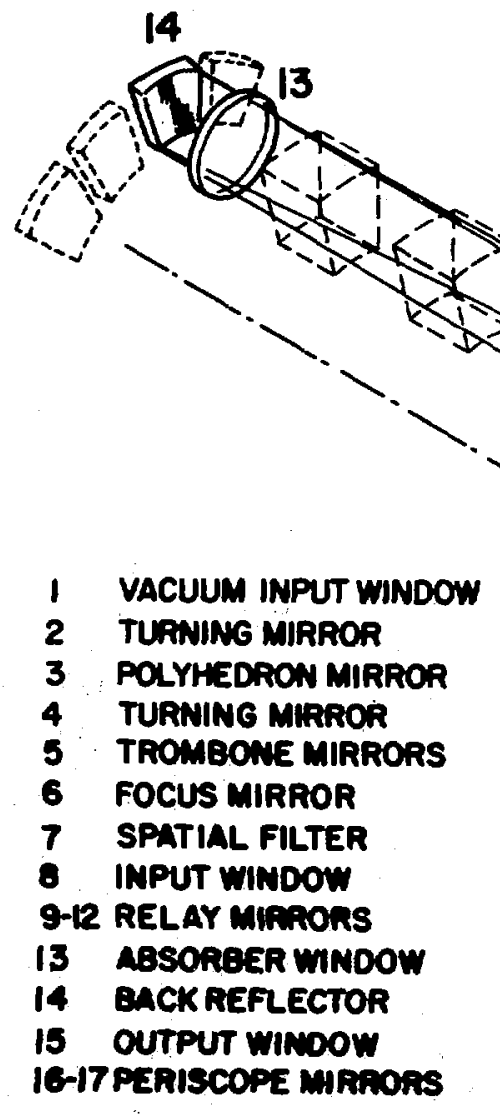

Fis. 8. Anteres power amplifier optical dements. 


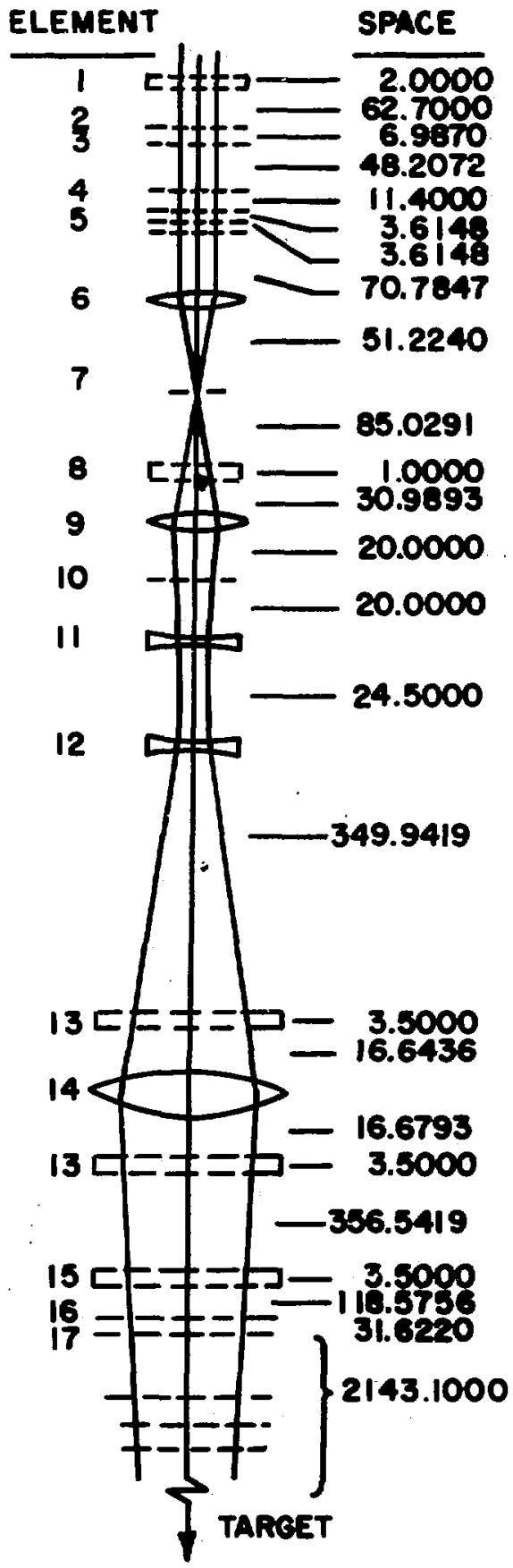

Fiz. 9. Optical elements spacing. The numbers refer to the same elements listed in Fis. 8. that is caused by laser beam impingenent could be formed at the spativi filter (element 7 in Fias. 8 and 9) in the following time sequences:

(1) $t=t_{0}=0 \mathrm{~ns}$ as the input beam pases the spatial filter on its way to the power amplifier;

(2) $t=t_{1}=9$ ns as part of the output beam leaving the power amplifier is intercepted by a relay mirror (element 12) and reaches the spatial filter uxing elements 8 through 11; and

(3) $t=t_{2}=537 \mathrm{~ns}$ as the retropulse returns from the fusion target to the spatial filter.

An edge fluence of approximately $45 \mathrm{~J} / \mathrm{cm}^{2}$ is required at time $\mathrm{t}_{\mathrm{b}}$ to obtain a beam blocking time of $50 \mathrm{~ns}$ (Fiz. 3). For an $f / 32$ airy disk pattern on the spatial filter at time $t_{\text {, }}$ the radii of the first and second minima are $0.4138 \mathrm{~mm}$ and $0.7474 \mathrm{~mm}$, respectively, for a ring area of $1.264 \mathrm{~mm}^{2}$. At an input energy of $7.0 \mathrm{~J}$ and an edge fluence of $46.6 \mathrm{~J} / \mathrm{cm}^{2}$, the first ring contains $8.4 \%$ of the input energy, yielding a plasma blocking time of $550 \mathrm{~ns}$ and an incident beam transmission of 75\%. An $\mathrm{f} / 32$ Gaussian beam profile at the spatial fitter at time $t_{6}$ has a theoretical beam radius at the $1 / \mathrm{e}^{2}$ intensity points of $0.2159 \mathrm{~mm}$ in the focal plane. This radius would provide sufficient edge fluence at the $0.3937-\mathrm{mm}$ radius spatial aperture for formation of a plasma. However, the plasma from the Gaussian beam would require further energy input at time $t_{1}$ to provide sufficient retropulse blocking at time $t_{2}$. At time $t_{1}$ the fluence reflected onto the spatial filter is less than $1.0 \mathrm{~J} / \mathrm{cm}^{2}$ in our configuration and would not appreciably affect the plasma density or beam blocking time. In Eqs. (2)(5), decreasing the space between the spatial filter and the focus mirror by 11.6 $\mathrm{mm}$ would produce an edge fhuence of $100 \mathrm{~J} / \mathrm{cm}^{2}$. This fluence would provide a beam blocking time of $820 \mathrm{~ns}$ relative to time $\dot{i}_{\mathbf{0}}$, a time greater than the retropulse return time $t_{2}$.

\section{v. CONCLUSIONS}

Laser-initiated plasma formation on or near metal surfaces has been the subject of considerable research, ${ }^{2-13}$ both in and out of a vacuum enviconment, using both short (1.0 ns) and lons (60-105 as) pulse widths. The longer pulse width ( $\leq 160 \mathrm{~ms}$ ) studies in air"-10 indicate that plasmas are initivted a local sites on the surface of the metali where defects or fialses exist that can be easily hested to reporization. Them planea is formed by surface-ewitud privies electrons beated by inverse-bremsetrablunes aborption in the presence of the 
neutral air molecules. The process results in initiation of a nonequilibrium air-breakdown cascade. In several experiments at 1.06 and $10.6 \mu \mathrm{m}$ where metal irises have been used in a vacuum environment, ${ }^{3,4,7}$ deterioration and enlargement of the spatial-filter aperture have occurred that are dependent on the incident energy. We also observed this phenomenon in our work, where stainless steel pinholes were initially used in a $10^{-6}$-torr vacuum. Aperture closure times of $\sim 1.0 \mathrm{~ns}$ were determined in several experiments, ${ }^{2-4,7}$ which are in agreement with data obtained in our experiments.

The Lif spatial filter seems most satisfactory for the Antares high-energy laser fusion system as a spatial filter and as a plasma shutter to isolate the laser system from retrop:lse damage with beam blocking times greater than thie retropulse return time. The LiF spatial filter is a passive device that functions in a vacuum environment with a high forward transmission and negligible backward transmission, using the incident laser-initiated plasma for retropulse blocking.

\section{ACKNOWLEDGMENTS}

The authors thank A. Saxman for providing invaluable advice, C. J. Elliott for valuable discussions. and M. Dugan for her help with data reduction.

\section{REFERENCES}

1. L. P. Bradley, E. L. Orham, I. F. Stowers, and P. Koert, "Development of a Plasma Retropulse Shutter for Shiva and Nova," Lawrence Livermore Laboratory report UCRL-52830 (September 1979).

2. S. J. Czuchlewski and J. F. Figueira, "Threshold Conditions for Laser-Initiated Plasma Shutters," Appl. Phys. Lett. 38, 325 (1981).

3. R. F. Benjamin, D. B. Henderson, K. D. Mitchell, M. A. Stroscio, and J. Thomas, "Laser-Target Retropulse Isolation Using an Aperture in Vacuum," Appl. Phys. Lett. 31, 511 (1977).
4. J. M. Averbach, N. C. Holmes, J. T. Hunt, and G. L. Linford, "Closure Phenomena in Pinholes Isradiated by Nd Laser Pulser," Appl. Opt. 18, 2495 (1979).

5. N. G. Loter, W. Halverson, and B. Lax, "Interaction of $\mathrm{CO}_{2}$ Laser Pulses with Solid Targets in Magnetic Fields," J. Appl. Phys. 52, 5014 (1981).

6. Roger B. Perkins and the Ineitial Fusion Program Staff, "Inertial Fusion Program, January 1-June 30, 1979," Los Alamos National Laboratory report LA-8114-PR (June 1981).

7. J. S. Pearlman and J. P. Anthes, "Closure of Pinholes Under Intense Laser Radiation," Appl. Opt. 16, 2328 (1977).

8. C. T. Walters, R. H. Barnes, and R. E. Beverly, III, "Initiation of Laser-Supported-Detonation (LSD) Waves," J. Appl. Phys. 49, 2937 (1978).

9. J. F. Figueira, S. J. Czuchlewski, C. R. Phipps, Jr., and S. J. Thomas, "Plasma-Breakdown Retropulse Isolators for the Infrared," Appl. Opt. 20, 838 (1981).

10. E. E. Bergmann, E. J. McLellan, and J. A. Webb, "Fast Nondamageable Laser-Pulse Detector Using Gaseous Plasma," Appl. Phys. Lett. 37, 18 (1980).

11. D. W. Forslund, J. M. Kindel, and K. Lee, "Theory of Hot-Electron Spectra at High Laser Intensity," Phys. Rev. Lett. 39, 284 (1977).

12. D. V. Giovanielli and R. P. Godwin, "Optics in Laser-Produced Plasmas,” Am. J. Phys. 43, 808 (1975).

13. J. S. Peariman and G. H. Dahlbacka, "Charge Separation and Target Voltages in Laser-Produced Plasmas," Appl. Phys. Lett. 31, 414 (1977). 
